Ferrata Storti Foundation

\section{Platelet glycoprotein VI and C-type lectin-like receptor 2 deficiency accelerates wound healing by impairing vascular integrity in mice}

\author{
Surasak Wichaiyo, ${ }^{1,2}$ Sian Lax, ${ }^{1}$ Samantha J. Montague, ${ }^{1} \mathrm{Zhi} \mathrm{Li},{ }^{3}$ \\ Beata Grygielska, ${ }^{1}$ Jeremy A. Pike,${ }^{4}$ Elizabeth J. Haining, ${ }^{1}$ Alexander Brill,,${ }^{1,5}$ \\ Steve P. Watson, ${ }^{1,4,6}$ and Julie Rayes ${ }^{1}$
}

Haematologica 2019

Volume 104(8):1648-1660

\section{Correspondence:}

JULIE RAYES

j.rayes@bham.ac.uk

STEVE P. WATSON

s.p.watson@bham.ac.uk

Received: October 12, 2018.

Accepted: January 28, 2019.

Pre-published: February 7, 2019.

doi:10.3324/haematol.2018.208363

Check the online version for the most updated information on this article, online supplements, and information on authorship \& disclosures: www.haematologica.org/content/104/8/1648

(C)2019 Ferrata Storti Foundation

Material published in Haematologica is covered by copyright. All rights are reserved to the Ferrata Storti Foundation. Use of published material is allowed under the following terms and conditions:

https://creativecommons.org/licenses/by-nc/4.0/legalcode. Copies of published material are allowed for personal or internal use. Sharing published material for non-commercial purposes is subject to the following conditions:

https://creativecommons.org/licenses/by-nc/4.0/legalcode, sect. 3. Reproducing and sharing published material for commercial purposes is not allowed without permission in writing from the publisher.
${ }^{1}$ Institute of Cardiovascular Sciences, College of Medical and Dental Sciences, University of Birmingham, Birmingham, UK; '2Department of Pharmacology, Faculty of Pharmacy, Mahidol University, Bangkok, Thailand; ${ }^{3}$ nstitute of Immunology and Immunotherapy, College of Medical and Dental Sciences, University of Birmingham, Birmingham, UK; ${ }^{4}$ Centre of Membrane Proteins and Receptors (COMPARE), Universities of Birmingham and Nottingham, The Midlands, UK; ${ }^{5}$ Department of Pathophysiology, Sechenov First Moscow State Medical University, Moscow, Russia and ${ }^{6}$ Institute of Microbiology and Infection, College of Medical and Dental Sciences, University of Birmingham, Birmingham, UK

\section{ABSTRACT}

latelets promote wound healing by forming a vascular plug and by secreting growth factors and cytokines. Glycoprotein (GP)VI and Ctype lectin-like receptor (CLEC)-2 signal through a (hem)-immunoreceptor tyrosine-based activation motif, which induces platelet activation. GPVI and CLEC-2 support vascular integrity during inflammation in the skin through regulation of leukocyte migration and function, and by sealing sites of vascular damage. In this study, we investigated the role of impaired vascular integrity due to GPVI and/or CLEC-2 deficiency in wound repair using a full-thickness excisional skin wound model in mice. Transgenic mice deficient in both GPVI and CLEC-2 exhibited accelerated skin wound healing, despite a marked impairment in vascular integrity. The local and temporal bleeding in the skin led to greater plasma protein entry, including fibrinogen and clotting factors, was associated with increased fibrin generation, reduction in wound neutrophils and M1 macrophages, decreased level of tumor necrosis factor (TNF)- $\alpha$, and enhanced angiogenesis at day 3 after injury. Accelerated wound healing was not due to developmental defects in CLEC-2 and GPVI double-deficient mice as similar results were observed in GPVI-deficient mice treated with a podoplanin-blocking antibody. The rate of wound healing was not altered in mice deficient in either GPVI or CLEC-2. Our results show that, contrary to defects in coagulation, bleeding following a loss of vascular integrity caused by platelet CLEC-2 and GPVI deficiency facilitates wound repair by increasing fibrin(ogen) deposition, reducing inflammation, and promoting angiogenesis.

\section{Introduction}

Cutaneous wound repair is a complex process, which requires a well-regulated interplay between diverse cell types and molecules. ${ }^{1}$ Four inter-related phases of wound healing have been described, namely hemostasis, inflammation, proliferation, and remodeling. ${ }^{1}$ Immediately upon injury, platelets and fibrin generate a hemostatic plug to prevent excessive blood loss. Additionally, the fibrin clot forms a scaffold to promote migration of local cells surrounding the wound. ${ }^{2}$ Deficiency in clotting factors, including tissue factor (TF), factor (F)VII or FIX, results in persistent intra-tissue bleeding and delayed wound healing.-5 Shortly after, neutrophils and inflammatory macrophages (M1) are recruited to eliminate microbes and cellular debris, driving the inflammatory phase. ${ }^{1,6}$ During the proliferative phase, re-epithelialization and angiogenesis promote cell growth and wound recovery. In addition, fibroblasts infiltrate the granulation tissue to produce extracellular matrix proteins, and to differentiate into myofibroblasts, mediating 
wound contraction. ${ }^{1}$ In parallel, the number of $\mathrm{M} 2$ reparative macrophages increases, contributing to resolution of inflammation. ${ }^{1,6}$ Complete wound closure and re-organization of collagen fibers restore skin integrity during the remodeling phase. ${ }^{1}$

Platelets play several roles in wound healing during the hemostatic, inflammatory, and vascular repair phases. ${ }^{7}$ Platelets secrete chemoattractants and growth factors that mediate cell recruitment and tissue repair, respectively. ${ }^{1,7}$ This is illustrated by the delay in healing of corneal epithelial abrasion in thrombocytopenic and P-selectindeficient mice. ${ }^{9}$ Moreover, platelet-rich plasma, which contains growth factors, promotes skin wound healing in mice $^{10}$ and is a possible therapeutic agent to facilitate wound repair.

Platelet immunoreceptor tyrosine-based activation motif (ITAM) receptors, GPVI and CLEC-2, share a common Src/Syk/PLC $\gamma 2$-dependent signaling pathway leading to platelet activation. ${ }^{11}$ A primary role for GPVI and a secondary role for CLEC-2 in maintaining vascular integrity in the inflamed skin has been demonstrated. ${ }^{12,13}$ In addition, CLEC-2 and GPVI are key regulators of inflammation. GPVI promotes a pro-inflammatory phenotype during glomerulonephritis, ${ }^{14}$ arthritis, ${ }^{15}$ and dermatitis. ${ }^{16}$ The CLEC-2-podoplanin axis is anti-inflammatory and protects against organ damage during lung and systemic inflammation. ${ }^{17,18}$

Due to the complex interplay between platelets and inflammation during wound healing, we hypothesize that GPVI and/or CLEC-2 regulate vascular integrity during wound repair and alter the healing process. In the present study, we show that deletion of both CLEC-2 and GPVI accelerates wound healing in a mouse model of fullthickness excisional skin wound repair. This is associated with a transient and self-limited bleeding (i.e. due to impaired vascular integrity), fibrin(ogen) matrix deposition, a reduction in wound neutrophils and M1 macrophages, and increased angiogenesis during the inflammatory phase. Taken together, we show that impaired vascular integrity-induced bleeding is beneficial during a model of sterile wound healing.

\section{Methods}

\section{Animals}

Male and female wild-type (WT), platelet-specific CLEC-2deficient (Clec1 $\left.b^{f / f} P f 4-C r e\right)$, GPVI knockout $\left(G p 6^{-/}\right)$, and CLEC2/GPVI double-deficient (Clect $b^{f l / f} P f 4-C r e / G p 6^{-1-}$; DKO) mice aged 8-10 weeks were used. All experiments were performed in accordance with UK laws (Animal Scientific Procedures Act 1986) with the approval of the local ethics committee and UK Home Office under PPL P0E98D513 and P14D42F37, respectively.

\section{Full-thickness excisional skin wound model}

A single full-thickness excisional skin wound was made on the shaved flank skin of mice using a $4 \mathrm{~mm}$-diameter biopsy punch (Kai Industries, Japan). Wounds were imaged using a Nikon COOLPIX B500 digital camera each day and wound size measured using calipers ${ }^{19}$ on a daily basis for up to nine days post injury. Wound area was calculated as described ${ }^{19}$ and presented as the percentage of initial wound size. ${ }^{10}$ In a second set of experiments, anti-podoplanin antibody (clone 8.1.1, 100 $\mu \mathrm{g})^{13,17,20}$ or IgG isotype control were intravenously injected 24 hours (h) before and again $24 \mathrm{~h}$ after wounding. Wound size was monitored for three days post injury.

\section{Other associated methods}

For details of other associated methods see the Online Supplementary Appendix.

\section{Results}

\section{CLEC-2 and GPVI ligands are present within perivascular areas during skin wounding}

In unchallenged WT mouse skin, collagen was abundantly expressed throughout the dermis and hypodermis, including around blood vessels, and podoplanin was predominantly expressed on lymphatic endothelial cells and on perivascular cells (Online Supplementary Figure S1A and $B)$. At day 3 post wounding, podoplanin expression was also observed surrounding blood vessels in WT and transgenic mice (Figure 1A). These podoplanin-expressing cells included pericytes (neuron-glial antigen 2; NG2+) (Figure $1 \mathrm{~A})$, fibroblasts (vimentin ${ }^{+}$), infiltrating monocytes $\left(\right.$ Ly6C $C^{+}$, and macrophages $\left(\mathrm{F} 4 / 80^{+}\right)$(Figure $\left.1 \mathrm{~B}\right)$. In addition, podoplanin was up-regulated on migrating keratinocytes, and on stromal and infiltrating cells within the granulation tissue (Online Supplementary Figure S1C). The level of perivascular podoplanin was increased in Clec1 $b^{f / f l} P f 4$-cre mice compared to WT mice (Figure 1A and $\mathrm{B})$. In DKO mice, podoplanin expression was similar to that observed in WT and $G p 6^{-1-}$ mice (Figure $1 \mathrm{~A}$ and B).

Three days after wound injury, platelets $\left(\mathrm{CD} 41^{+}\right)$were observed in close proximity to the vessel wall (surrounded by pericytes) in WT, Clec1 $b^{f / f} \mathrm{Pf} 4$-cre, and $\mathrm{Gpb}^{-1-}$ mice (Figure 1A, arrow). In DKO mice, platelets were more widely distributed, including at the vessel wall and in the surrounding tissue (Figure 1A, star). The platelet count in $\mathrm{Clec} \mathrm{b}^{f / f} \mathrm{Pf} 4$-cre and DKO mice was approximately $25 \%$ and $40 \%$ lower than in WT, respectively (Online Supplementary Figure S1D) and was not altered by wound injury.

These results demonstrate that platelets are present in perivascular areas during the initial wound healing process in mouse skin where the ligands for GPVI and CLEC-2 are also observed. Deletion of CLEC-2 from platelets is associated with increased podoplanin-expressing cells in the perivascular space during wound healing while concurrent ablation with GPVI reverses this phenotype.

\section{Cutaneous wound healing is accelerated in mice deficient in platelet CLEC-2 and GPVI}

To determine the contribution of platelet ITAM receptors in wound repair, we monitored the time course of wound closure in WT and platelet ITAM receptor(s)-deficient mice. All mouse strains exhibited complete wound closure within nine days post injury (Figure 2A). However, mice deficient in both GPVI and CLEC-2 demonstrated accelerated repair (Figure 2B), characterized by the presence of a dark scab and redness around the wound (Figure 2A), especially within the first three days after injury compared to WT and single ITAM-deficient mice. There was no discernible difference in wound appearance in any of the mouse strains after four days post injury (Figure 2A) and there was no difference in macroscopic wound size at day 9 post injury (Figure 2B). 
However, the morphometric analysis of skin histology at this time point revealed a significantly smaller scar in DKO mice, as shown by a shorter length of hyperplastic epidermis compared to WT and Clec1 $b^{f / f} \mathrm{Pf} 4$-cre mice (Figure 2C and D) and a narrower inter-subcutaneous gap than WT mice (Figure 2C and E).

At day 3 post injury, re-epithelialization was observed in all groups (Figure 3A). However, this process was enhanced in DKO mice compared to WT and Clec1 $b^{f / f} \mathrm{Pf4}$ cre mice, but not $\mathrm{Gpb}^{-1}$ mice (Figure $3 \mathrm{~B}$ ). There was no sig- nificant difference in wound contraction between all tested groups (Figure $3 \mathrm{C}$ ). DKO mice also had a larger area of granulation tissue compared to WT mice (Figure $3 \mathrm{~A}$ and D). Improved wound healing was associated with enhanced angiogenesis as assessed by the increase in $\mathrm{CD} 1^{+}$area in DKO animals at day 3 post injury (Figure $3 \mathrm{E}$ and $\mathrm{F}$ ), although the density of blood vessels $\left(\mathrm{CD} 31^{+}\right)$ and lymphatic vessels (podoplanin ${ }^{+}$) in unchallenged skin was similar among all groups (Online Supplementary Figure $S 1 E$ and $F)$.
A
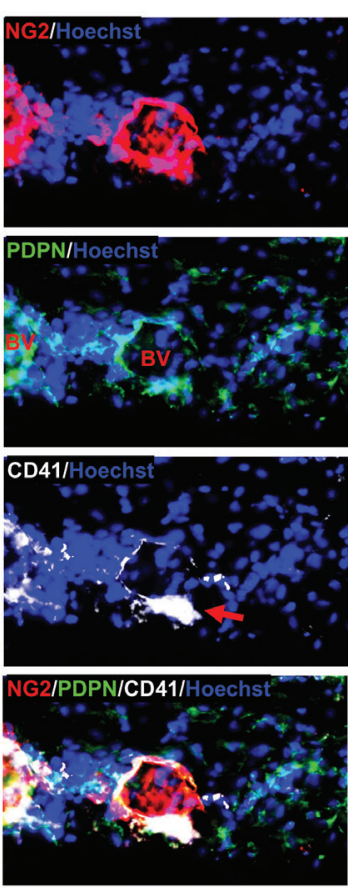

B
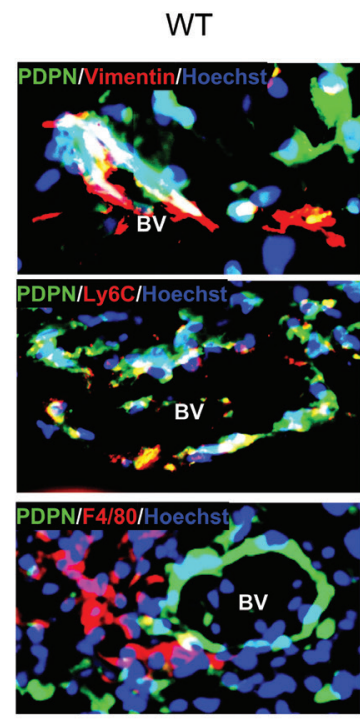

Clec1 $b^{f / f f l} P f 4-c r e$
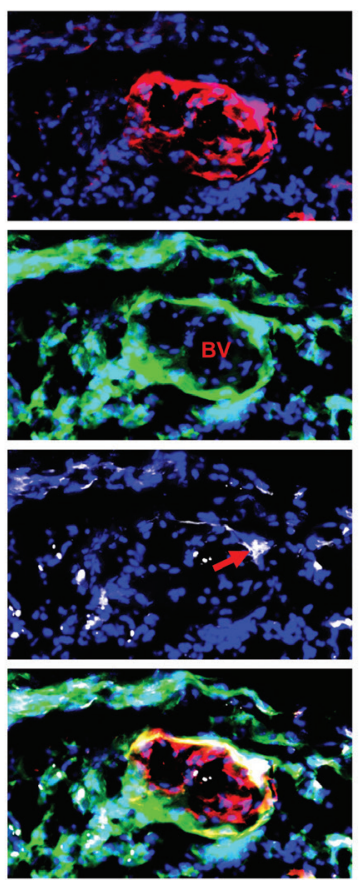

Clec $1 b^{f l f f l} P f 4-c r e$
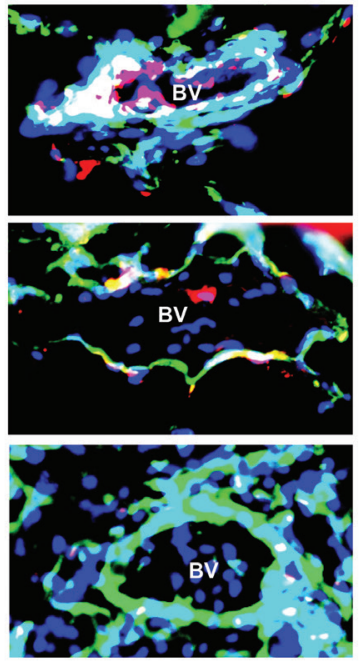

Gp6-1-
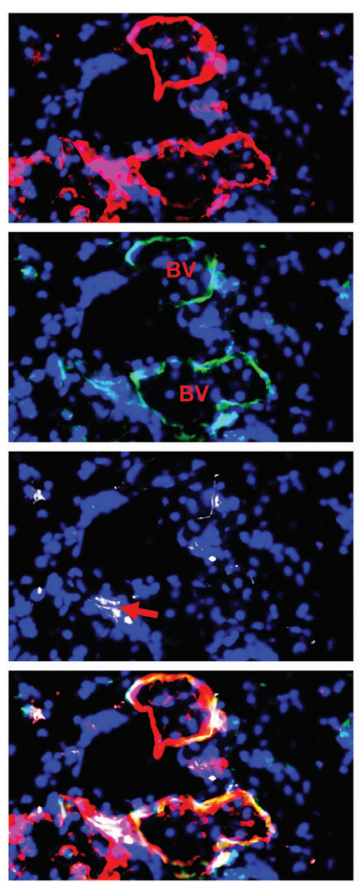

Gp6--
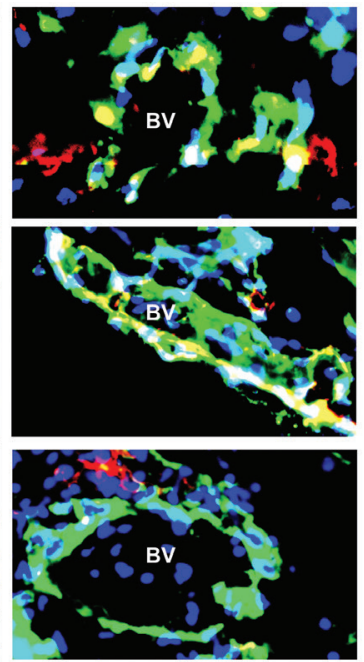

DKO
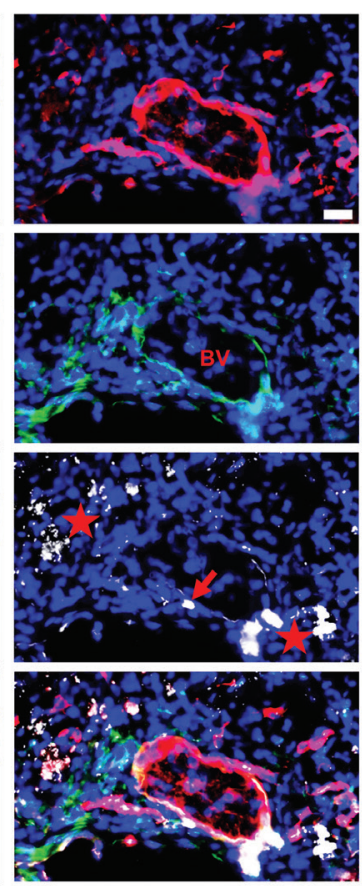

DKO

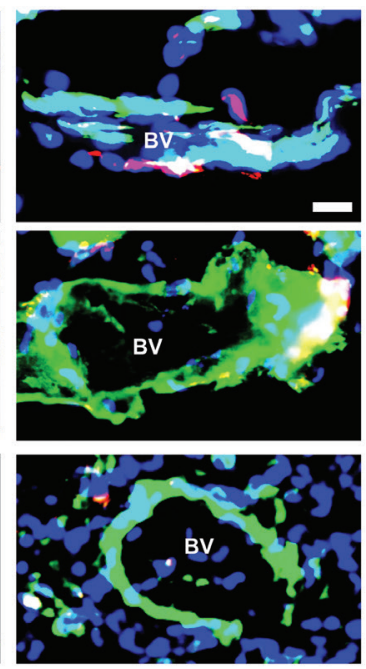

Figure 1. Podoplanin-expressing cells are present at perivascular area in contact with platelets during skin wound repair. (A) Immunofluorescence staining of NG2 (red), podoplanin (green) and CD41 (white) illustrates platelets and podoplanin-expressing pericytes (NG2+) around blood vessel at day 3 after injury ( $\mathrm{n}=5-7$ ). Hoechst counterstains nuclei (blue). Arrow points to platelets at perivascular site. Star indicates extravascular localization of platelets. Scale bar=20 $\mu \mathrm{m}$. (B) Podoplanin (green) was double-stained with either vimentin (red; top) or Ly6C (red; middle) or F4/80 (red; bottom), which are located around blood vessel (surrounded by NG2 pericytes) at day 3 after injury ( $n=4-5)$. Scale bar=20 $\mu \mathrm{m}$. BV: blood vessel. 
These results demonstrate that deletion of both CLEC2 and GPVI accelerates skin wound closure, enhances reepithelialization and angiogenesis, and reduces scar formation.

GPVI and CLEC-2 maintain vascular integrity during the inflammatory phase of wound repair

The redness surrounding the wound in DKO mice in the inflammatory phase is indicative of increased vascular leakiness. Macroscopic examination of the skin at day 3 post injury demonstrated vasodilation and bleeding into the wound as well as into the surrounding skin in DKO mice, with less severe vascular leakage seen in $\mathrm{Gp}^{-1-}$ mice (Figure 4A). Hematoxylin \& Eosin (H\&E) staining confirmed the extravasation of red blood cells (RBC) in the dermis at the edge of the wound at day 3 post injury in both DKO and $\mathrm{Gp}^{-\%}$ mice (Online Supplementary Figure $S 2 A)$. Clearance of extravascular RBC was observed at day 9 post injury in all groups (Online Supplementary Figure $S 2 B)$. Due to blood/lymphatic mixing phenotype in $\mathrm{DKO}$, and to a lesser degree in Clec1 $b^{f / l} \mathrm{Pf} 4$-cre mice, $\mathrm{RBC}$ were also present in lymphatic vessels (podoplanin ${ }^{+}$) (Online Supplementary Figure S2C).

These observations demonstrate marked impairment of vascular integrity during the inflammatory phase of wound repair in DKO mice, with a milder phenotype in $\mathrm{Gpb}^{-1}$ mice. Vascular leakage is diminished in later phases when inflammation subsides.

\section{GPVI and CLEC-2 deficiency increases fibrin(ogen) deposition during the inflammatory phase of repair} Increased vascular permeability results in leakage of
A

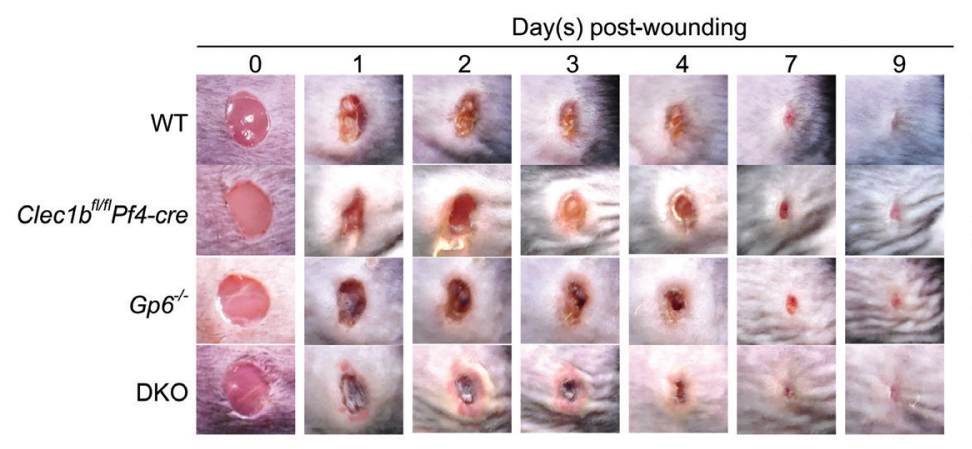

B

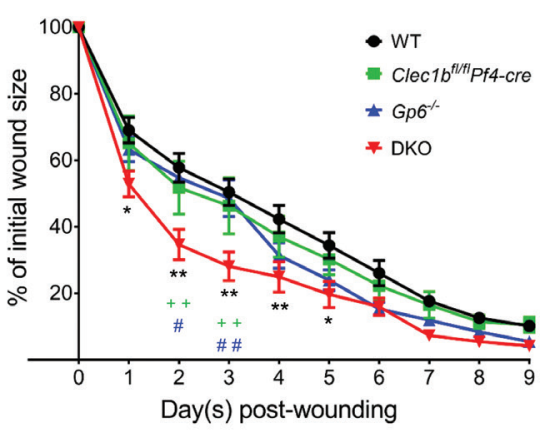

C
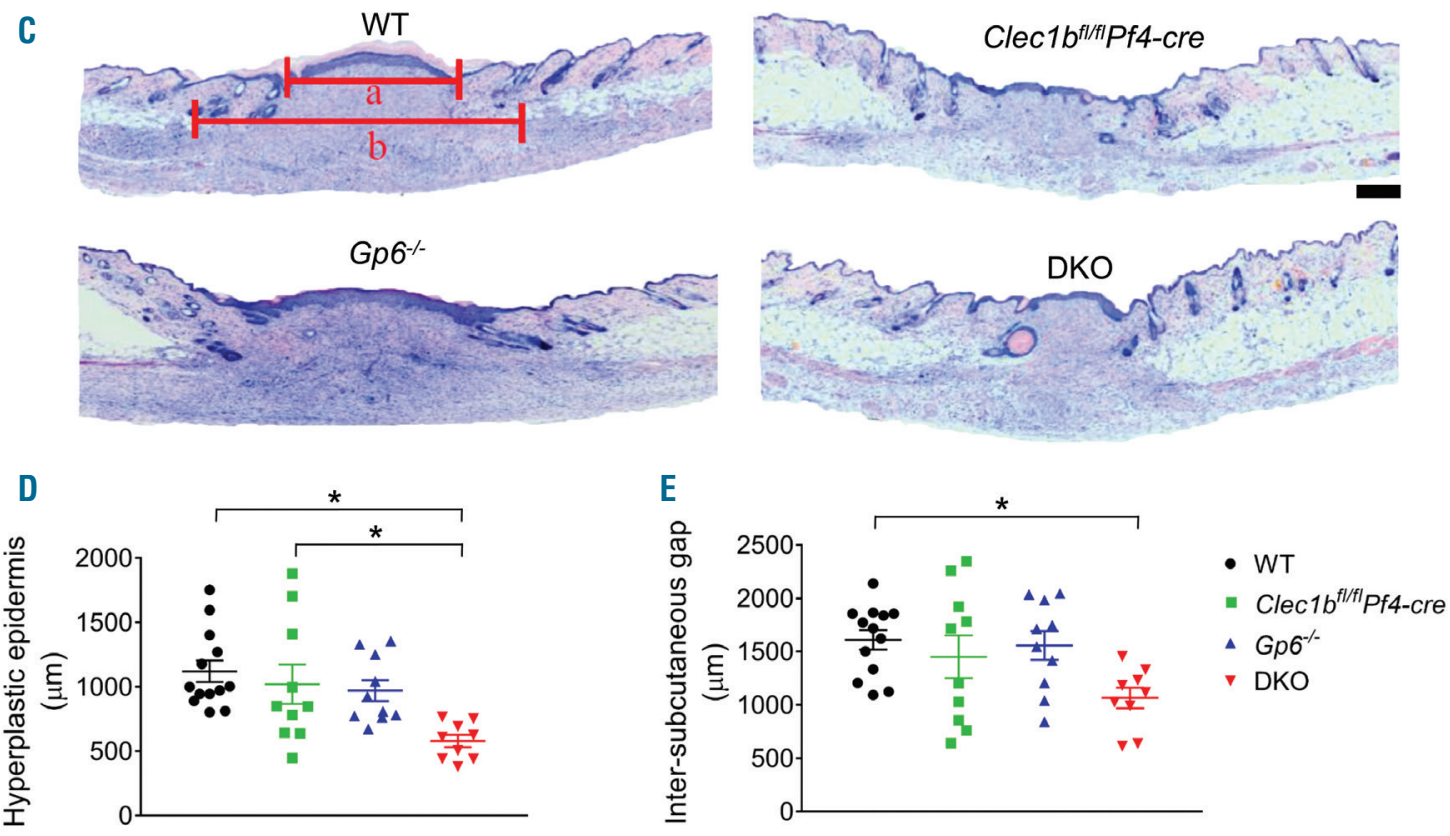

Figure 2. Deletion of platelet immunoreceptor tyrosine-based activation motif (ITAM) receptors accelerates skin wound repair process. Mice were subjected to a full-thickness excisional skin wound and wound closure was monitored for nine days after injury. (A) Macroscopic appearance of wound at indicated time points. (B) Changes of wound size over nine days post injury $(n=10-13)$. (C) Hematoxylin \& Eosin staining at day 9 post-injury $(n=9-13)$. a: length of hyperplastic epidermis; b: inter-subcutaneous distance. Scale bar=200 um. (D) Measurement of the length of hyperplastic epidermis. (E) Measurement of inter-subcutaneous distance. All graphs are presented as mean \pm Standard Error of Mean (SEM). Kinetics of wound closure (B) are analyzed by two-way ANOVA with Bonferroni's multiple comparison test. ${ }^{*} P<0.05$; ${ }^{*} P<0.01$. ${ }^{*}$ WT versus DKO. ${ }^{+}$Clec $1 b^{\sharp / f} P f 4$-cre versus DKO. ${ }^{*} G p 6{ }^{-/}$versus DKO. Other parameters are analyzed by one-way ANOVA with Bonferroni's multiple comparison test. $* P<0.05$. 
blood cells and plasma proteins into the wound ${ }^{21}$ where they come in contact with $\mathrm{TF}^{5,22}$ which activates the extrinsic pathway of blood coagulation. An increase in fibrinogen deposition was particularly marked in the granulation tissue at day 3 post injury (Figure $4 \mathrm{~B}$ and $\mathrm{C}$ ) with no significant alteration in TF expression in DKO mice (Online Supplementary Figure S3A and B). Fibrin con- tent in the wound scab was similar between WT and $\mathrm{DKO}$ mice at day 1 post injury (Online Supplementary Figure S3C and D), but was significantly increased in the DKO mice at day 3 post injury, compared to WT and Clec1 $b^{f / / 7} \mathrm{Pf} 4$-cre mice but not to $\mathrm{Gp} 6^{-/}$mice, which exhibited a more moderate vascular leakage (Figure 4D and E). At day 9 post injury, fibrin was mainly located on the

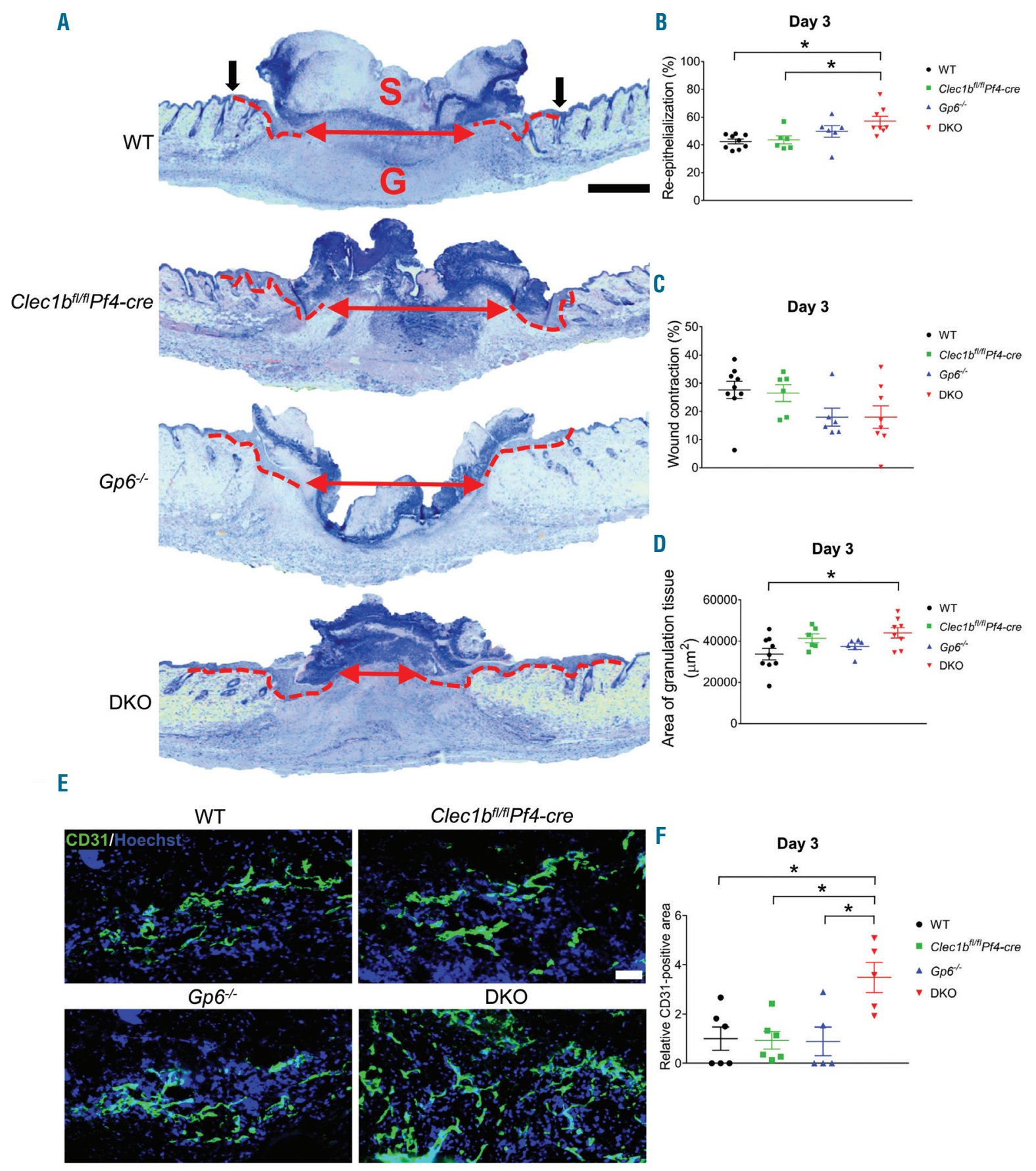

Figure 3. Enhanced re-epithelialization and angiogenesis occur at the early phase of wound healing in the absence of GPVI and CLEC-2. (A) Hematoxylin \& Eosin staining at day 3 post-injury $(n=6-9)$. Dotted line indicates hyperplastic coverages. Black arrow points to wound edge. Red arrow indicates gap between epithelial tongues. S: scab; G: granulation tissue. Scale bar=500 um. (B) Measurement of re-epithelialization. (C) Measurement of wound contraction. (D) Quantification of granulation tissue area. (E) Detection of endothelial cells (CD31 ${ }^{+}$cells; green) in wound area at day 3 post injury. Hoechst counterstains nuclei (blue). Scale bar=50 $\mu \mathrm{m}$. (F) Quantification of $C D 31^{+}$area within the wound at day 3 post injury $(n=5-6)$. Graphs are presented as mean \pm Standard Error of Mean and analyzed by oneway ANOVA with Bonferroni's multiple comparison test. $* P<0.05$. 
upper part of the scar in all groups (Figure 4F) and was notably lower in the DKO mice compared to WT and $\mathrm{Gp}^{-1}$ mice (Figure 4G). There was no significant difference in wound (myo)fibroblasts (Online Supplementary Figure $S 3 E-G$ ) and collagen content (Online Supplementary Figure $\mathrm{S} 3 \mathrm{H}$ and $I$ ) between DKO mice and WT at day 3 and day 9 post injury

These results indicate that the loss of vascular integrity during the inflammatory phase increases extravasation of plasma proteins, including fibrinogen and clotting factors, into the wound of DKO mice. The increase in fibrinogen and fibrin deposition is associated with accelerated

WT

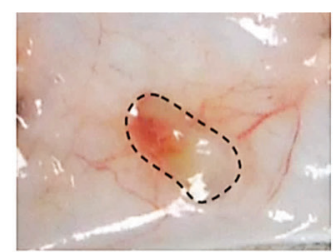

WT

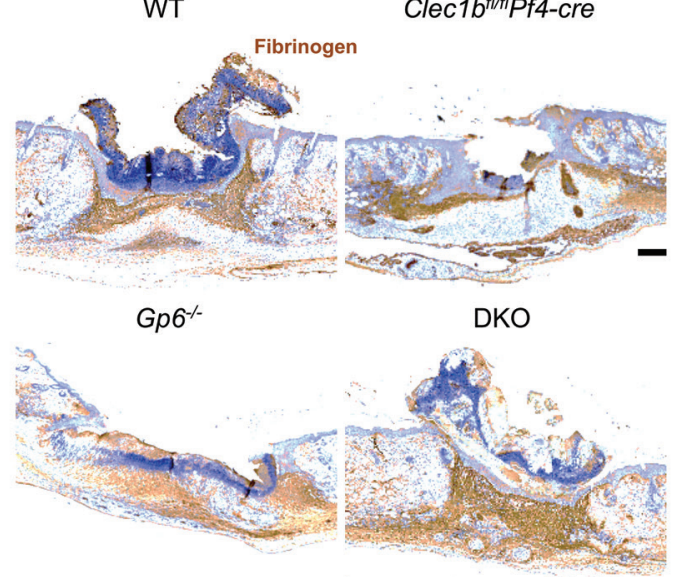

D

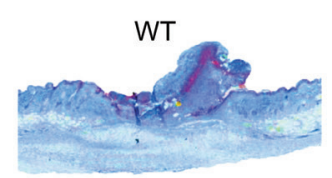

$G p 6^{--}$

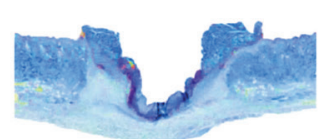

$\mathbf{F}$

WT

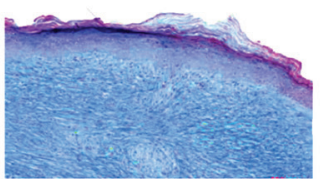

$\mathrm{Gp}^{-1}$

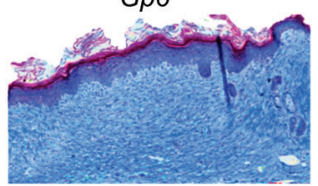

Clec1 $1 b^{f / f t} P f 4-c r e$

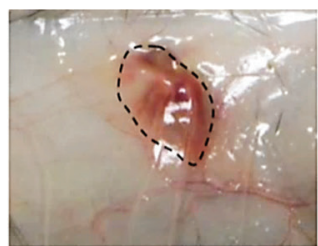

Clec1 $b^{f / f i} P f 4-c r e$

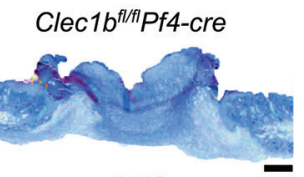

DKO

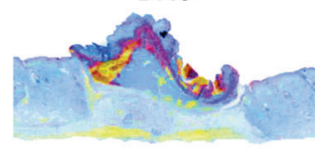

Clec1 $1 b^{f / f t} P f 4-c r e$

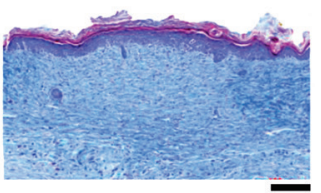

DKO

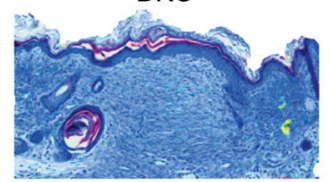

$\mathrm{Gp} 6^{-1-}$
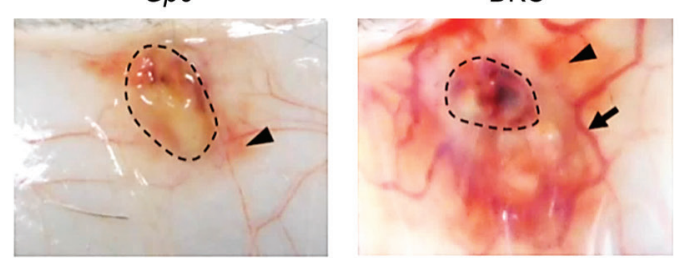

C

Day 3

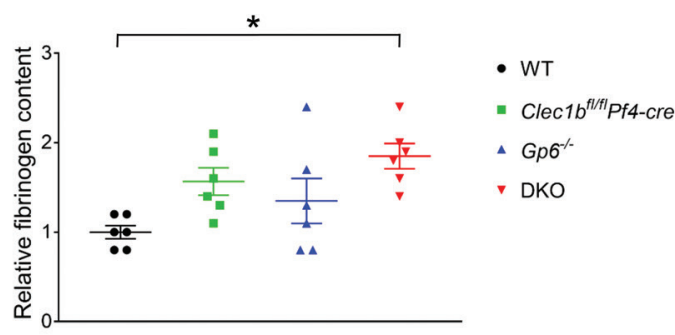

E

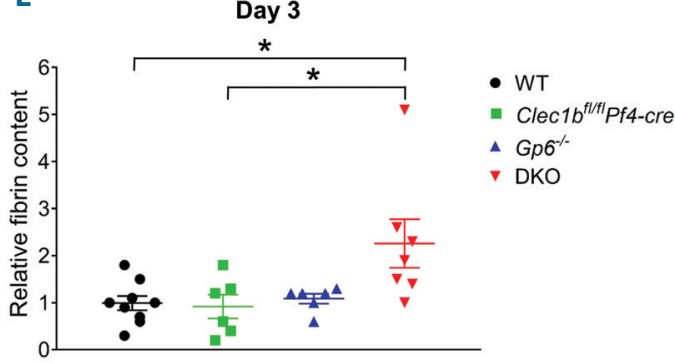

G

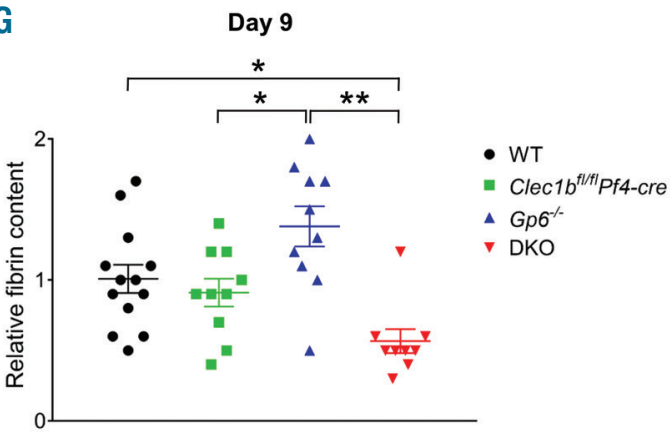

Figure 4. Lack of platelet immunoreceptor tyrosine-based activation motif (ITAM) receptors causes local and temporal bleeding leading to fibrin(ogen) deposition during inflammatory phase of wound repair. (A) Macroscopic images of inner side of skin wound at day 3 post injury ( $n=4-6)$. Dotted circle indicates wound area. Arrow points to dilated vessel. Arrowhead shows bleeding into surrounding skin. (B) Fibrinogen staining (brown) of skin wound at day 3 post injury. (C) Quantification of fibrinogen content at day 3 post injury $(n=6)$. (D) Martius scarlet blue (MSB) staining of skin wound at day 3 post-injury. Red: old fibrin; blue: collagen; yellow: red blood cells/fresh fibrin. (E) Quantification of fibrin content in the wound at day 3 post injury ( $n=6-9)$. (F) MSB staining of wound scar at day 9 post injury. (G) Quantification of fibrin content in the scar at day 9 post injury ( $n=9-13)$. Graphs are presented as mean \pm Standard Error of Mean and analyzed by one-way ANOVA with Bonferroni's multiple comparison test. $* P<0.05 ; * * P<0.01$. Scale bar $=200 \mu \mathrm{m}$. 
wound healing and is then cleared from the healing wound.

\section{Platelet immunoreceptor tyrosine-based activation motif receptor deficiency reduces wound neutrophils and M1 macrophages during the inflammatory phase}

The influx of inflammatory cells was investigated at days 1,3 , and 9 post injury. Neutrophil $\left(\mathrm{Gr}-1^{+}\right)$infiltration on day 1 post injury was similar between WT and DKO mice (Online Supplementary Figure S4A and B). At day 3 post injury, DKO mice showed a significant impairment in neutrophil infiltration compared to WT and Clec $1 b^{n / p} P f 4$-cre mice but not Gp $6^{-1}$ mice (Figure $5 \mathrm{~A}$ and B). The decrease in neutrophil infiltration was confirmed using anti-Ly6G antibody clone 1A8 (data not shown). A 2fold increase in wound neutrophils was observed in WT but not in DKO mice at this time relative to day 1 post injury (Figure 5C). At day 9 post injury, DKO mice showed higher numbers of wound neutrophils than WT (Figure 5D and E), although neutrophil level was significantly decreased in both groups relative to day 1 and day 3 post injury (Figure 5C). Blood neutrophil counts were similar in unchallenged mice across all groups (Figure 5F). At day 3 post injury, the number of blood neutrophils was significantly decreased in WT and Clec $1 b^{f / f} P f 4-c r e$ (Figure 5F) while remaining unaltered during the time course of wound healing in DKO and $\mathrm{Gp}^{-/}$mice (Figure $5 \mathrm{~F}$ ). The decrease in neutrophil infiltration was not due to a defect of chemoattractants at the wound site as measured by the presence of chemokine CXCL-1 (Online Supplementary Figure S6A and B) or platelet factor 4 (PF4) (Online Supplementary Figure S6C and D). In vitro migration towards N-formyl-methionyl-leucyl-phenylalanine (FMLP) using bone marrow-derived neutrophils demonstrated that migration of neutrophils from WT mice was
A

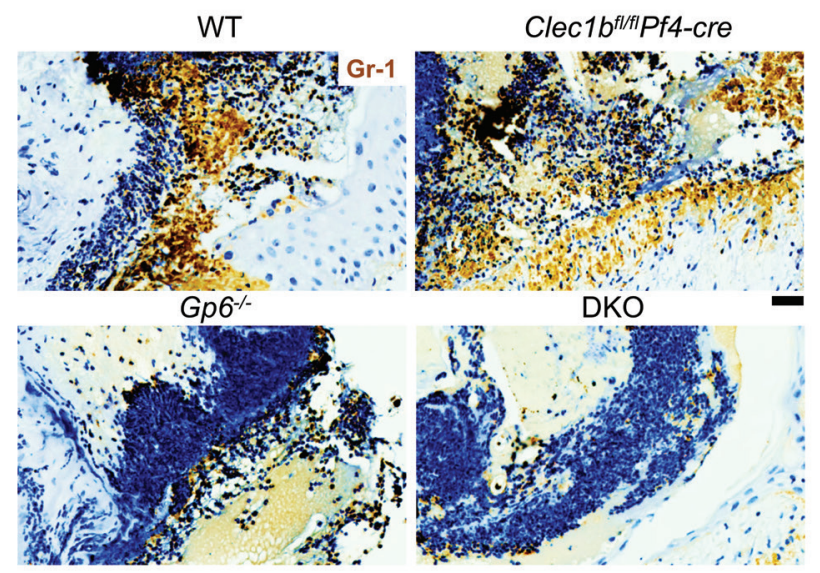

D

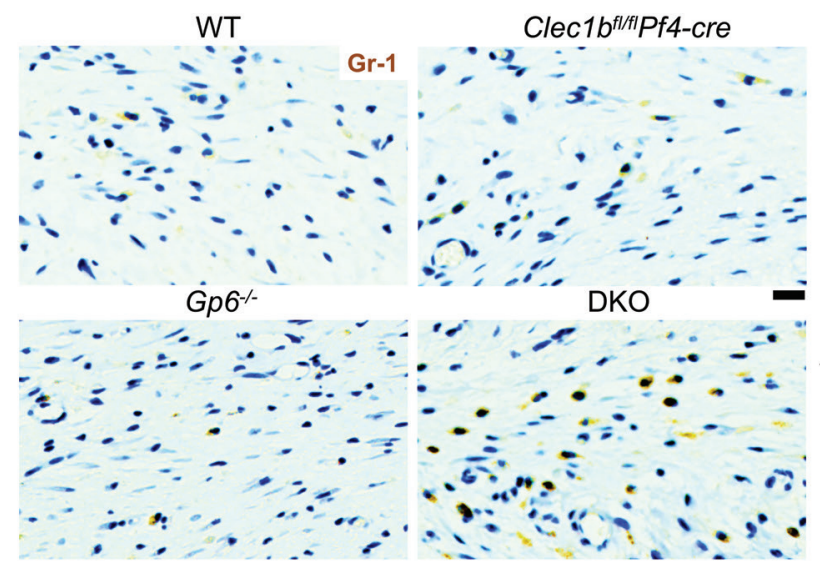

B

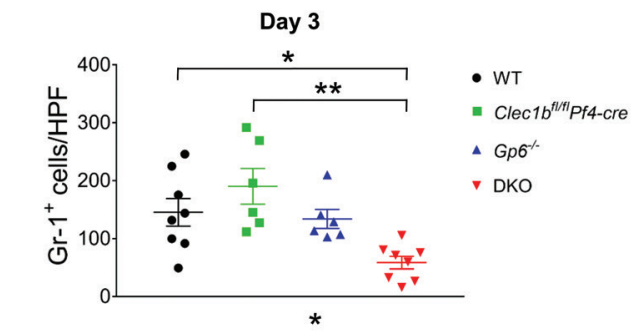

C
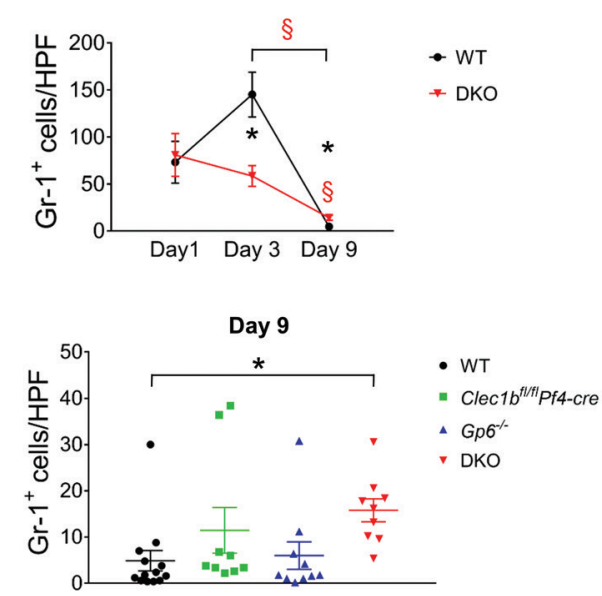

$\mathbf{F}$

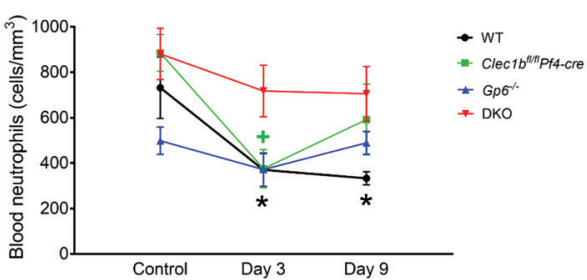

Figure 5. Neutrophil influx is decreased during the inflammatory phase of wound healing following platelet CLEC-2 and GPVI double-deletion. (A) Detection of neutrophils (Gr-1 staining; brown) in wound at day 3 post injury. (B) Quantification of neutrophils (Gr- $1^{+}$cells) in wound at day 3 post injury. $* P<0.05$; $* \star P<0.01$. (C) Comparison of $\mathrm{Gr}^{+}{ }^{+}$cells between day 1 , day 3 , and day 9 post injury in wild-type (WT) and DKO mice. $P<0.05$ in *WT and ${ }^{5}$ DKO mice, compared to the data at day 1 post injury, respectively. The bracket shows $P<0.05$ for the comparison between day 3 and day 9 post injury in *WT and ${ }^{\circledR} D K O$ mice, respectively. (D) Detection of neutrophils (Gr-1 staining; brown) in wound at day 9 post injury. (E) Quantification of neutrophils (Gr- $1^{+}$cells) in wound at day 9 post injury. $* P<0.05$. (F) Comparison

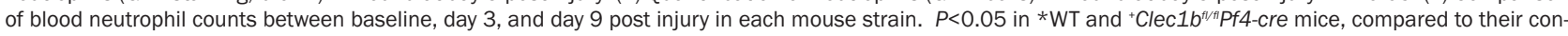
trol, respectively. Sample numbers in unchallenged control $=10$, day $1=5$, day $3=6-9$, and day 9 post injury $=9-13$, respectively. Graphs are presented as mean \pm Standard Error of Mean and analyzed by one-way ANOVA with Bonferroni's multiple comparison test. Scale bar $=20 \mu \mathrm{m}$. 
attenuated by fibrinogen, and strongly inhibited by crosslinked fibrin compared to the migration through collagen (Online Supplementary Figure S7). Fibrinogen and fibrin showed a similar degree of inhibition for the migration of neutrophils from DKO mice (Online Supplementary Figure S7).

There was no significant difference in wound monocytes $\left(\right.$ Ly6C $C^{+}$) at day 1 post injury between DKO mice and WT (Online Supplementary Figure S4C and D). At day 3 post injury, DKO mice showed a significantly higher number of wound monocytes compared to all other strains (Figure 6A and B), which was reflected by a 4-fold increase in wound monocytes relative to day 1 post injury (Figure 6C). At day 9 post injury, monocytes within the wound remained at a low level in WT and single-knockout mice (Figure 6D and E). Wound monocytes in DKO mice were decreased at this time compared to day 3 post injury (Figure 6C), but remained higher than other groups
(Figure 6D and E). In blood, Clec1 $b^{f / f l} \mathrm{Pf} 4$-cre exhibited an increase in baseline circulating monocytes compared to WT and DKO mice (Online Supplementary Figure S4G, left). Blood monocytes were greatly reduced at day 3 and day 9 post injury in all groups compared to their unchallenged controls (Figure 6F). However, the level of blood monocytes in DKO mice was significantly higher than Clec1 $1 b^{1 / f l} P f 4$-cre mice at day 3 post injury (Online Supplementary Figure $S 4 G$, middle) and then all groups at day 9 post injury (Online Supplementary Figure S4G, right).

The influx of macrophages $\left(\mathrm{F} 4 / 80^{+}\right)$in $\mathrm{DKO}$ mice was reduced at day 1 post injury compared to WT (Online Supplementary Figure $S 4 E$ and F). At day 3 post injury,

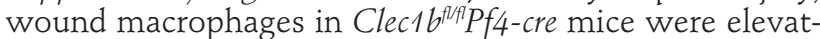
ed, compared to all other strains (Figure 7A and B). A 6fold increase in wound macrophages was observed in WT mice at day 3 relative to day 1 post injury (Figure 7C). DKO mice showed a significant reduction in
A

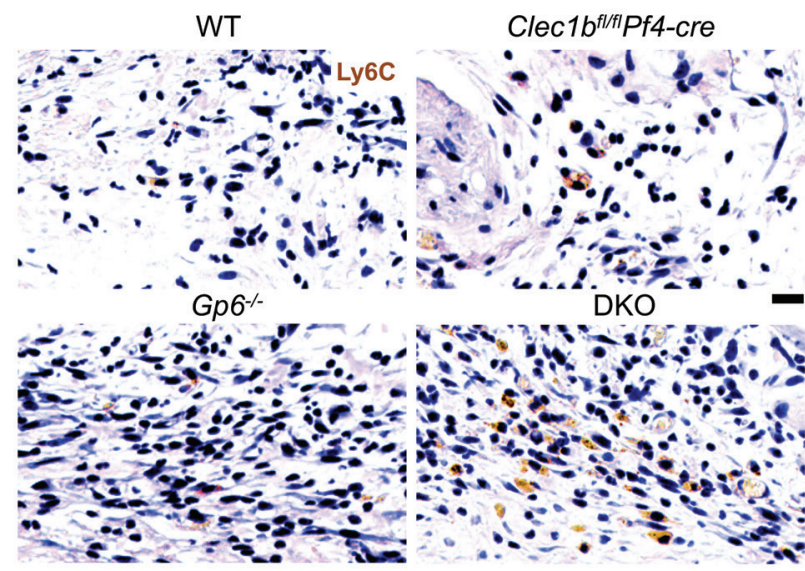

D

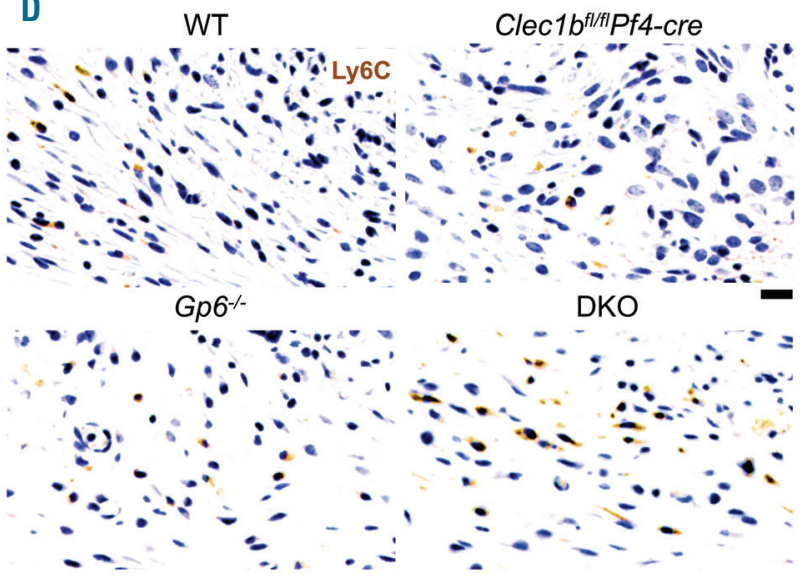

B

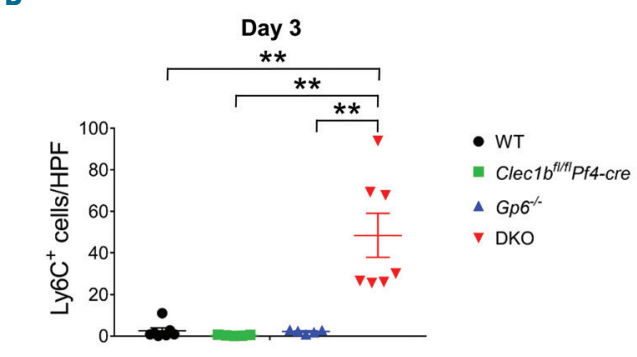

C

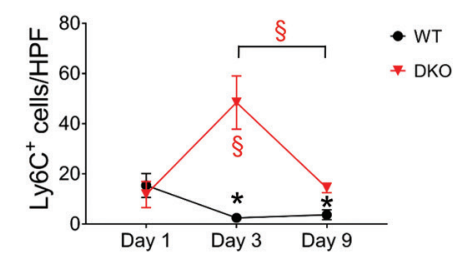

E

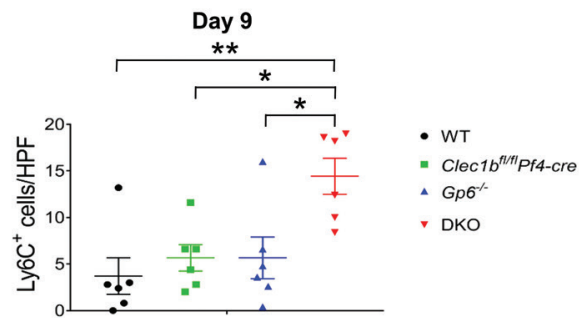

$\mathrm{F}$

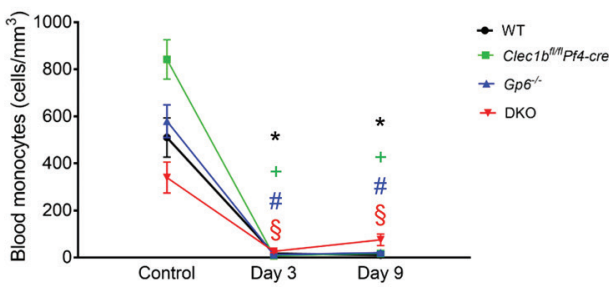

Figure 6. A higher number of wound monocytes is observed during the inflammatory phase of repair in mice that lack both GPVI and CLEC-2. (A) Detection of monocytes (Ly6C $C^{+}$cells; brown) in wound at day 3 post-injury. (B) Quantification of Ly6C ${ }^{+}$cells in wound at day 3 post-injury ( $n=5-7$ ). $* * P<0.01$. (C) Comparison of Ly $6 \mathrm{C}^{+}$cells between day 1 , day 3 , and day 9 post-injury in WT and DKO mice. The symbols * and $\S$ indicate $P<0.05$ in WT and DKO mice, compared to the data at day 1 post injury, respectively. The bracket shows $P<0.05$ for the comparison between day 3 and day 9 post injury in ${ }^{\S} \mathrm{DKO}$ mice. (D) Detection of Ly6C $\mathrm{C}^{+}$cells (brown) in wound at day 9 post injury. (E) Quantification of Ly6C $\mathrm{C}^{+}$cells in wound at day 9 post injury $(\mathrm{n}=6)$. $* P<0.05$; $* * P<0.01$. (F) Comparison of blood monocyte counts between baseline, day 3, and day 9 post injury in each mouse strain. The symbols *, +, \#, and § indicate $P<0.05$ in WT, Clec1 $b^{f / f} P f 4-c r e, ~ G p 6 \%$, and DKO mice, compared to their control, respectively. Sample numbers in unchallenged control, $n=10$; day $1, n=5$; day 3, $n=6-9$; day 9 post injury, $n=10-13$, respectively. Graphs are presented as mean \pm Standard Error of Mean and analyzed by one-way ANOVA with Bonferroni's multiple comparison test. Scale bar $=20 \mu \mathrm{m}$. 
macrophages compared to WT but not $G p 6^{-1-}$ mice at this time (Figure $7 \mathrm{~B}$ and $\mathrm{C}$ ). Inducible nitric oxide synthase (iNOS)-expressing macrophages (M1 phenotype) (Figure $7 \mathrm{D}$ and $\mathrm{E}$ ) and TNF- $\alpha$ level (Figure $7 \mathrm{G}$ and $\mathrm{H}$ ) within the wound of DKO mice were decreased whereas Fizz-1positive macrophages (a M2 marker) were similar to WT
(Online Supplementary Figure S5D and E) at day 3 post injury. At day 9 post injury, wound macrophages in DKO mice were similar to WT and Clec1 $b^{f / f} \mathrm{Pf} 4$-cre mice (Online Supplementary Figure S5D and E) with no difference in M1 (Figure 7D and $\mathrm{F}$ ) and $\mathrm{M} 2$ macrophages (Online Supplementary Figure S5A and C) between WT and DKO
A

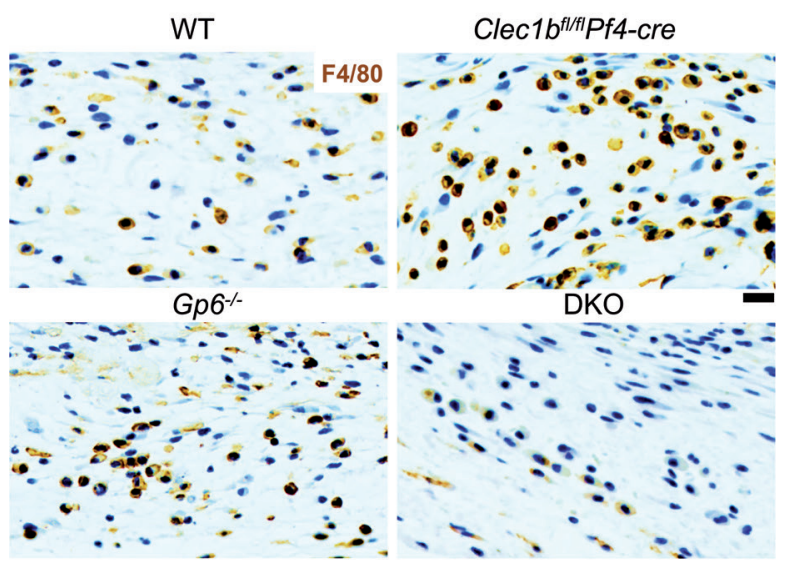

D
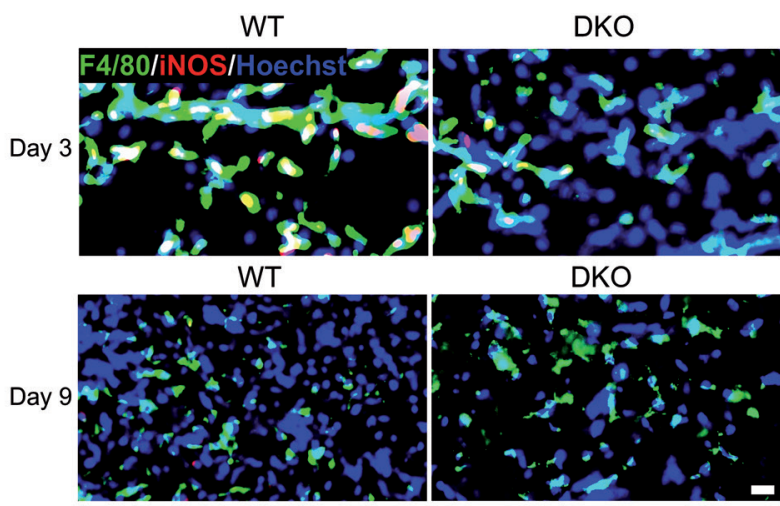

DKO

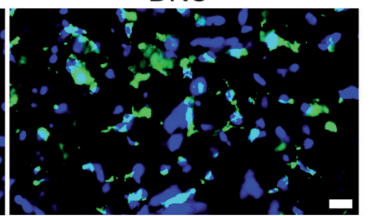

G

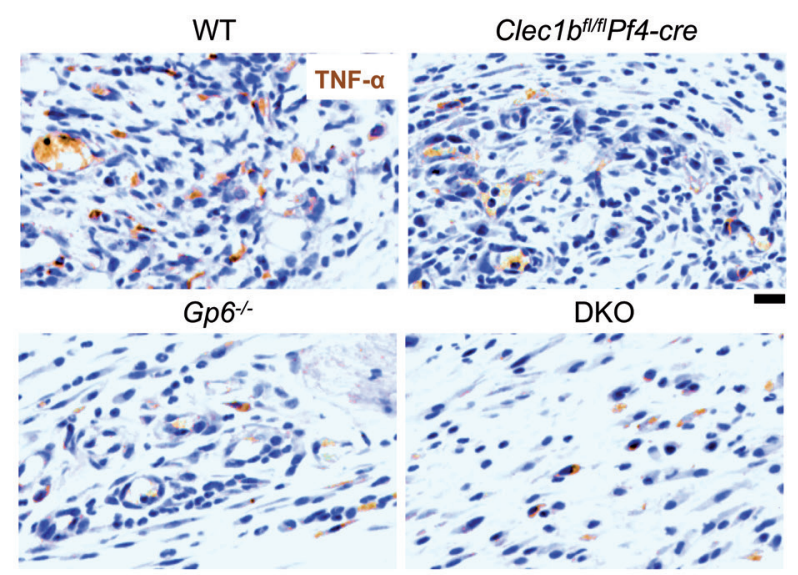

B

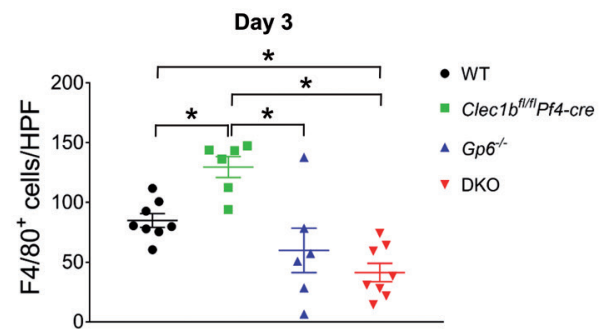

C

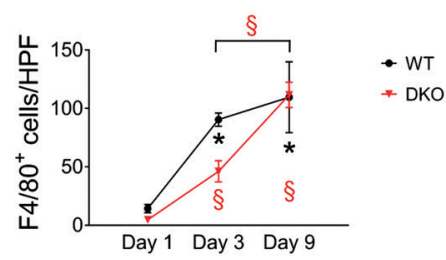

E

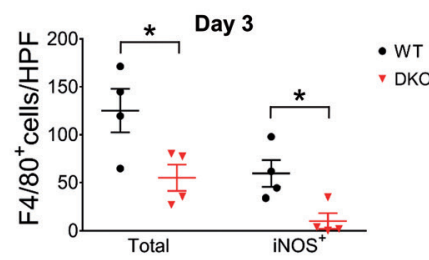

$\mathrm{F}$

H
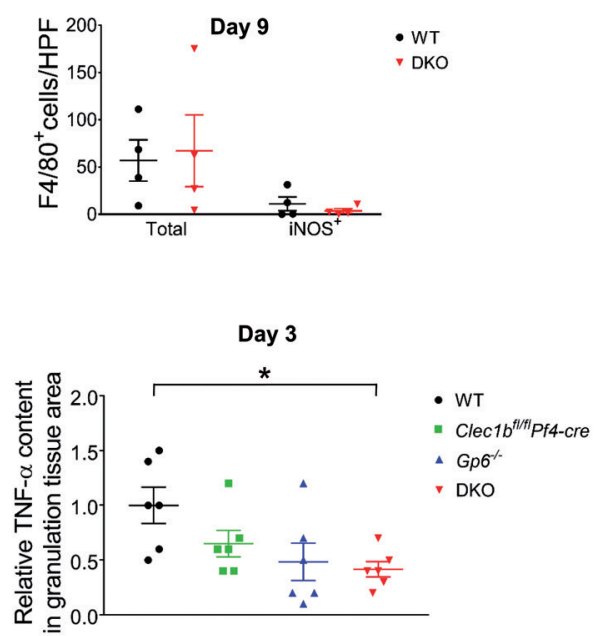

Figure 7. M1 macrophages and TNF- $\alpha$ level are reduced during the inflammatory phase of wound healing in platelet immunoreceptor tyrosine-based activation motif (ITAM) receptor-deficient mice. (A) Detection of macrophages (F4/80+ cells; brown) in wound at day 3 post injury. (B) Quantification of $\mathrm{F} 4 / 80^{+}$cells in wound at day 3 post injury $(n=6-8)$. $* P<0.05 ; * * P<0.01$. (C) Comparison of $F 4 / 80^{+}$cells between day 1 , day 3 , and day 9 post injury in wild-type (WT) and DKO mice. The symbols * and $\S$ indicate $P<0.05$ in WT and DKO mice, compared to the data at day 1 post injury, respectively. The bracket shows $P<0.05$ for the comparison between day 3 and day 9 post injury in ${ }^{5}$ DKO mice. Sample numbers at days post injury: at day 1, n=5; day 3, n=6-9; day 9 , n=10-13, respectively. (D) Immunofluorescence double staining of iNOS (red) and F4/80 (green) in the wound of WT and DKO mice at day 3 ( $n=4)$ and day 9 ( $n=4)$ post injury. Hoechst counterstains nuclei (blue). (E) Quantification of M1 macrophages (iNOS ${ }^{+} F 4 / 80^{+}$cells; yellow) at day 3 post injury ( $\left.n=4\right)$ ) *P<0.05. (F) Quantification of M1 macrophages (iNOS ${ }^{+}$F4/80+ cells; yellow) at day 9 post injury $(n=4)$. (G) Immunohistochemistry staining of TNF- $\alpha$ (brown) in the wound at day 3 post injury. (H) Quantification of TNF- $\alpha$ level in granulation tissue area at day 3 post injury $(n=6)$. ${ }^{*}<<0.05$. Graphs are presented as mean \pm Standard Error of Mean and analyzed by either Student $t$-test $(\mathrm{E}, \mathrm{F})$ or one-way ANOVA with Bonferroni's multiple comparison test (B, C, and $\mathrm{H})$. Scale bar $=20 \mu \mathrm{m}$. 
strains. $G p 6^{-1}$ mice showed a lower number of macrophages within the wound scar than in Clec1 $b^{\text {fl/f }} \mathrm{Pf4}$ cre mice at day 9 post injury (Online Supplementary Figure S5E).

Overall, these data illustrate that CLEC-2 deletion promotes leukocyte sequestration in the wound during the inflammatory phase, especially macrophages. Deletion of both ITAM receptors leads to a significant reduction in wound neutrophils and M1 macrophages during this phase and a decrease in TNF- $\alpha$ expression in the tissue.
Inhibition of CLEC-2-podoplanin axis accelerates wound healing in GPVI-deficient mice

Clec1 $b^{f / f l} \mathrm{Pf} 4$-cre and DKO mice present with blood/lymphatic mixing and moderate thrombocytopenia. ${ }^{11,17}$ To investigate the influence of these defects on wound healing, we injected $\mathrm{Gp}^{-1}$ mice with an antibody to podoplanin (anti-podoplanin-treated $\mathrm{Gp}^{-/}$mice) that blocks CLEC-2-podoplanin interaction. A significant acceleration in wound healing was observed in antipodoplanin-treated $\mathrm{Gp}^{-/}$mice at day 2 and day 3 post
A

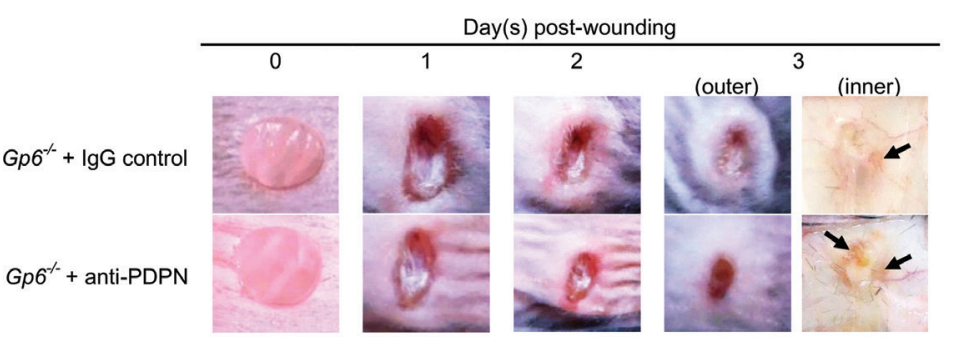

B

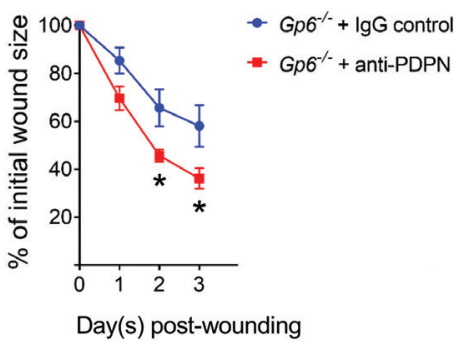

Gp6 $6^{--}+$anti-PDPN

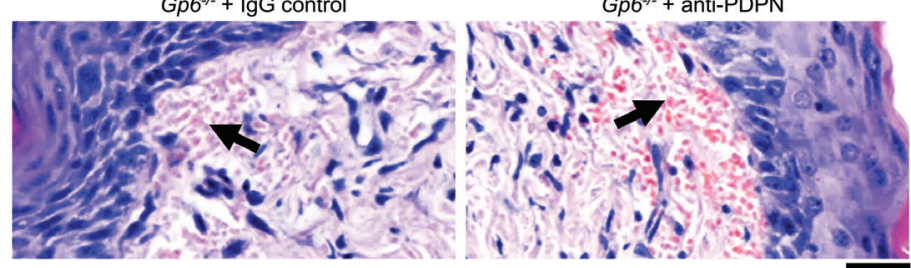

D

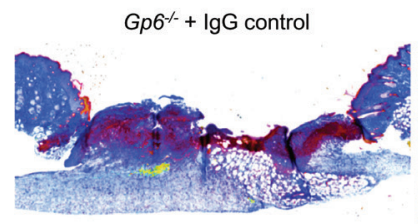

$\mathbf{F}$

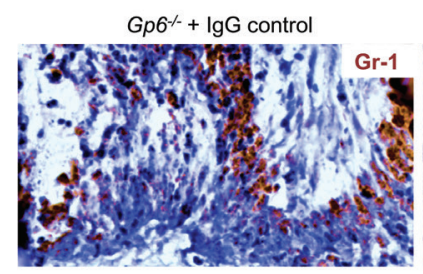

Gp6 $6^{-}+$anti-PDPN

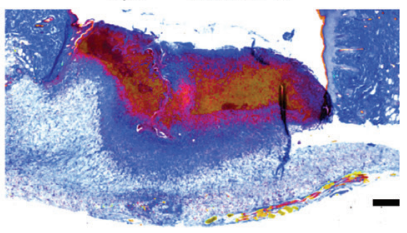

Gp6\% + anti-PDPN

E

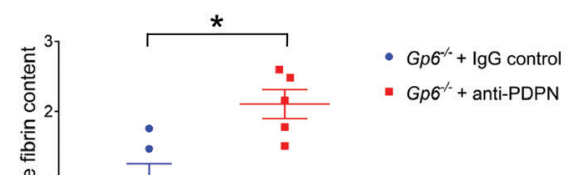

G
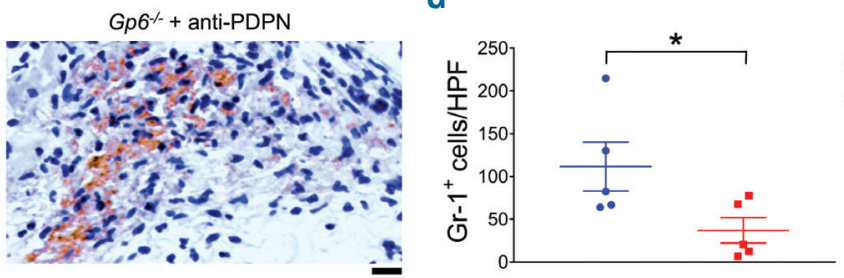

- $\mathrm{Gp6}^{-\alpha}+\lg \mathrm{G}$ control

- $G p 6^{-\alpha}+$ anti-PDPN

H
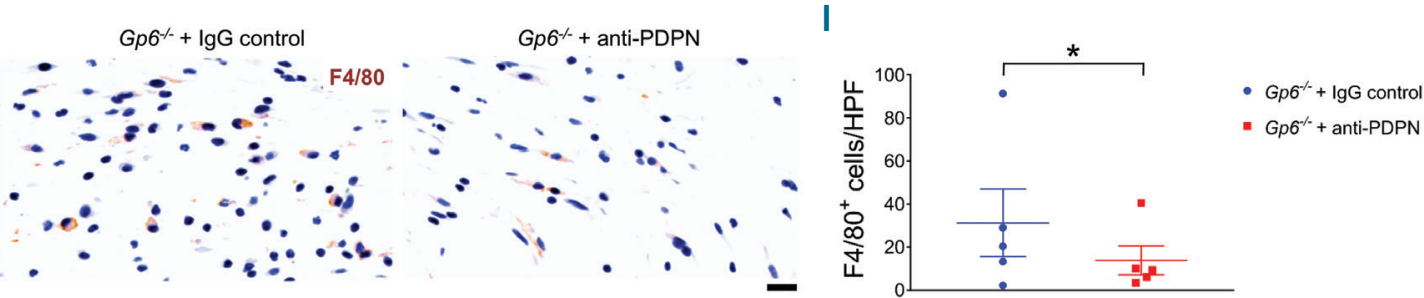

Figure 8. Anti-podoplanin antibody injection in Gp6 mice (Gp6 + anti-PDPN) simulates the accelerated phenotype of skin wound repair observed in DKO mice. (A) Macroscopic appearance of wound at indicated time points. Arrow points to intra-skin bleeding around the wound at day 3 post injury. (B) Changes of wound size over 3 days post injury $(n=5)$. (C) Hematoxylin \& Eosin staining at day 3 post-injury $(n=5)$. Arrow points the bleeding into surrounding skin. Scale bar $=20 \mu$ m. (D) Martius scarlet blue staining of skin wound at day 3 post injury. Red: old fibrin, blue: collagen, yellow: red blood cells/fresh fibrin. Scale bar $=200$ um. $(E)$ Quantification of fibrin content (red) in the wound at day 3 post injury ( $n=5$ ). (F) Staining of neutrophils (Gr-1; brown) in wound area at day 3 post injury. (G) Quantification of neutrophils (Gr- $1^{+}$cells) in wound area at day 3 post injury $(n=5)$. (H) Detection of macrophages ( $F 4 / 80$ staining; brown) in wound area at day 3 post injury. (I) Quantification of macrophages ( $44 / 80^{+}$cells) in wound area at day 3 post-injury ( $n=5$ ). All graphs are presented as mean \pm Standard Error of Mean. Kinetics of wound closure $(B)$ are analyzed by two-way ANOVA with Bonferroni's multiple comparison test. Other parameters are analyzed by Student $t$-test. * $P<0.05$. 
injury (Figure 8A and $\mathrm{B}$ ), in association with enhanced vascular leakage (Figure $8 \mathrm{~A}$ and $\mathrm{C}$ ) and increased fibrin deposition (Figure 8D and E). Moreover, decreased wound neutrophils (Figure 8F and G) and macrophages (Figure $8 \mathrm{H}$ and $\mathrm{I}$ ), and higher wound monocytes (Online Supplementary Figure $S 8 A$ and $B$ ) but unaltered level of TNF- $\alpha$ (Online Supplementary Figure S8C and D) were observed in anti-podoplanin-treated $\mathrm{Gp}^{-1}$ mice. The reepithelialization (Online Supplementary Figure S8E and F), granulation tissue formation (Online Supplementary Figure $S 8 E$ and $H$ ), and angiogenesis (Online Supplementary Figure $S 8 I$ and $J$ ) were also increased in anti-podoplanin-treated $\mathrm{Gp}^{-/}$mice. However, no significant change was seen in wound contraction (Online Supplementary Figure S8E and $G)$. Similar to DKO mice, extravasation of platelets was detected together with the presence of anti-podoplanin antibody on pericytes and other perivascular cells in antipodoplanin-treated $\mathrm{Gp}^{-\%}$ mice at this time (Online Supplementary Figure S9).

These data indicate that the accelerated wound healing in DKO mice is not due to developmental defects or thrombocytopenia, but due to a combined loss of the interaction of platelet CLEC-2 and GPVI with their respective ligands.

\section{Discussion}

In this study, we show that combined deletion of platelet CLEC-2 and GPVI promotes healing of a fullthickness skin wound in mice compared to WT or singleknockout mice. The accelerated wound closure is accompanied by impairment of vascular integrity, rapid reepithelialization and an increase in granulation tissue formation, resulting in a smaller wound scar. This is also associated with elevated levels of fibrinogen and fibrin in the tissue, reduced infiltration of leukocytes, and enhanced angiogenesis. A proposed model for the multifactorial regulation of accelerated wound healing in the absence of the two ITAM receptors is shown in Online Supplementary Figure S10.

Wound healing is a multistep process involving coagulation, vascular permeability changes, inflammation, cell proliferation, and cell migration. ${ }^{1,221}$ In our study, we show that deletion of CLEC-2 and GPVI in platelets leads to increased bleeding into the wound, in association with accelerated wound repair. These effects are not due to blood/lymphatic vessel mixing or the reduction in platelet count in the DKO mice as similar results were observed in anti-podoplanin-antibody treated $\mathrm{Gp}^{-1-}$ mice, which do not have these defects. Acceleration of wound healing is also observed in mice treated with histamine $e^{23}$ or serum fraction of the natural latex from rubber tree, ${ }^{21}$ which enhances vascular permeability, a milder form of vascular leakage. Therefore, impaired vascular integrity as seen in the DKO platelets or increased permeability leads to extravasation of growth factors, cytokines, and plasma proteins to the tissue, and promotion of wound healing.

The role of blood coagulation in wound repair has been studied in mice expressing low $\mathrm{TF}^{5}$ or lacking FIX (hemophilia $B$ mice). ${ }^{3,5}$ In these two models, the deficiency in clotting factors leads to a persistent hematoma formation (due to a prolonged subcutaneous bleeding) and a reduction in fibrin generation, both of which contribute to a delay in wound repair. Chronic hemorrhage also con- tributes to the characteristics of non-healing wounds in cancers, which promote tumor growth. ${ }^{24-26}$ In contrast, we show that in the presence of an intact coagulation cascade, the transient and self-limited bleeding into the wound (which is rich in $T^{5,22}$ ) caused by the impairment of vascular integrity at an initial stage, is associated with accelerated wound repair. This is most likely due to increased entry of platelets, clotting factors, and plasma proteins, leading to fibrinogen accumulation and fibrin generation. Thus, the mechanism of bleeding determines whether it is beneficial or detrimental to wound repair.

Fibrinogen and fibrin are not only crucial for clot formation, but also act as a natural suture/sealant, providing a matrix for cell migration as well as a reservoir for growth factors and cytokines. ${ }^{2}$ For example, fibrin exposes plasminogen to migrating keratinocytes, which in turn convert plasminogen into plasmin to mediate fibrinolysis, allowing the cells to move along the fibrin(ogen) matrix. ${ }^{27}$ ${ }^{29}$ Similarly, endothelial cells proliferate and migrate over fibrin(ogen), forming a capillary tube that contributes to angiogenesis during wound healing. ${ }^{30-32}$ In DKO mice, we therefore propose that fibrin(ogen) accumulation promotes re-epithelialization and angiogenesis, and accelerates wound repair.

In contrast, fibrinogen ${ }^{33}$ and a high concentration of fibrin $^{34}$ inhibit neutrophil migration as previously reported and in line with our in vitro data. These data may explain our observations that in DKO mice, wound neutrophils at day 3 did not increase over the amount seen at day 1 post injury. This is in contrast to wound neutrophil accumulation in WT, which was higher at day 3 than at day 1 post injury. Increased fibrin content in the wound was also observed in DKO mice compared to WT at day 3 post injury. Therefore, the physical obstruction and anti-migratory properties of the fibrin clot is likely to inhibit neutrophil wound entry observed in DKO mice. Moreover, given that, at this early phase, there was no significant difference in the level of blood neutrophils between WT and $\mathrm{DKO}$ mice, altered neutrophil turnover/apoptosis in $\mathrm{DKO}$ mice is unlikely to make a significant contribution to the reduction in wound neutrophils observed in DKO compared to WT at day 3 post injury.

Consistent with our data, several lines of evidence demonstrate improved wound healing in neutrophildepleted conditions. ${ }^{35-38}$ Indeed, neutrophil infiltration mediates damage through a production of proteases, ${ }^{35}$ oxidative radicals, elastase ${ }^{39}$ and neutrophil extracellular traps, ${ }^{37}$ all of which can delay the healing process. A similar effect of fibrin(ogen) might also apply to monocyte/macrophage recruitment since fibrinogen has an anti-adhesive effect against monocytes. ${ }^{40} \mathrm{~A}$ recent sterile wound model has demonstrated that $\mathrm{Ly} 6 \mathrm{C}^{+}$monocytes are present throughout wound healing and differentiate into M1 macrophages during inflammatory phase and $\mathrm{M} 2$ macrophages during the reparative phase. ${ }^{41}$ In addition, it has been shown that TNF- $\alpha$ secretion is increased during monocyte-to-M1-macrophage differentiation. $^{41,42}$ The reduction in TNF- $\alpha$ during the inflammatory phase is associated with the increase in wound monocytes but not macrophages. This suggests an inhibition in monocyte-to-M1-macrophage transition in $\mathrm{DKO}$ mice, resulting in a significant change in immune cell infiltration in the wound.

Depletion of macrophages in the first five days has previously been shown to delay the later stages of wound 
closure, but not during the inflammatory phase (day 13). ${ }^{43}$ This is due to a decrease in M2 macrophages, ${ }^{43}$ supporting our observation that a reduction in wound macrophages, particularly M1 phenotype, in the early phase does not negatively affect wound closure but may contribute to the reduction in scar formation. ${ }^{43}$ The alteration in M2 macrophages was not observed in DKO mice although the previous dermatitis model has reported an increase number of $\mathrm{M} 2$ phenotypes in GPVI-deficient mice, ${ }^{16}$ suggesting other contributing factors for macrophage polarization during skin wound healing. ${ }^{6}$

The increased risk of wound contamination is a concern in the context of intra-tissue bleeding and reduced wound leukocytes. However, it has recently been shown that rapid formation of a fibrin film over the surface of the wound is protective against bacterial infection. ${ }^{44}$ This process might also reduce the need for leukocyte infiltration to kill microbes. Moreover, a previous study has reported that a 2-fold increase in wound neutrophils is driven by Staphylococcus aureus infection. ${ }^{45}$ Whether the beneficial potential of targeting GPVI and CLEC-2 might modulate the risk of wound infection requires further investigation.

In conclusion, we show that deletion of platelet GPVI and CLEC-2 facilitates cutaneous wound repair through a local and temporal vascular leakage leading to increased fibrin(ogen) deposition and reduced leukocyte infiltration. Thus, impaired vascular integrity due to the loss of GPVI and CLEC-2 is beneficial to wound repair. This contrasts with results in coagulation-deficient mice, with differences explained by altered formation of fibrin and most likely alteration in immune cell trafficking. A shorter duration of healing lowers the risk of complications (e.g. infection) and the cost of caring for the wound. ${ }^{46}$ Based on our study, targeting CLEC-2 and GPVI at the wound site together with optimal wound care (e.g. aseptic dressing) might represent a new pathway to promote healing and reduce scar formation.

\section{Acknowledgments}

The authors would like to thank the BMSU at the University of Birmingham for technical support in animal experiments and the Technology Hub for imaging assistance.

\section{Funding}

This work was supported by the Ministry of Sciences and Technology of Thailand and the British Heart Foundation (RG/13/18/30563). SPW holds a BHF Chair (CHO3/003).

\section{References}

1. Shaw TJ, Martin P. Wound repair at a glance. J Cell Sci. 2009;122(Pt 18):3209 3213.

2. Drew AF, Liu H, Davidson JM, Daugherty CC, Degen JL. Wound-healing defects in mice lacking fibrinogen. Blood. 2001;97(12):3691-3698.

3. Hoffman M, Harger A, Lenkowski A, Hedner U, Roberts HR, Monroe DM. Cutaneous wound healing is impaired in hemophilia B. Blood. 2006;108(9):30533060 .

4. $\mathrm{Xu} \mathrm{Z,} \mathrm{Xu} \mathrm{H,} \mathrm{Ploplis} \mathrm{VA,} \mathrm{Castellino} \mathrm{FJ.}$ Factor VII deficiency impairs cutaneous wound healing in mice. Mol Med. 2010; 16(5-6):167-176.

5. Monroe DM, Mackman N, Hoffman M. Wound healing in hemophilia B mice and low tissue factor mice. Thromb Res. 2010;125 Suppl 1:S74-77.

6. Sindrilaru A, Scharffetter-Kochanek K Disclosure of the Culprits: MacrophagesVersatile Regulators of Wound Healing. Adv Wound Care (New Rochelle). 2013; 2(7):357-368.

7. Golebiewska EM, Poole AW. Platelet secretion: From haemostasis to wound healing and beyond. Blood Rev. 2015;29(3):153162 .

8. Brill A, Elinav H, Varon D. Differential role of platelet granular mediators in angiogenesis. Cardiovasc Res. 2004;63(2):226-235.

9. Li Z, Rumbaut RE, Burns AR, Smith CW Platelet response to corneal abrasion is necessary for acute inflammation and efficient re-epithelialization. Invest Ophthalmol Vis Sci. 2006;47(11):4794-4802.

10. Yang HS, Shin J, Bhang SH, et al. Enhanced skin wound healing by a sustained release of growth factors contained in platelet-rich plasma. Exp Mol Med. 2011;43(11):622629.

11. Bender M, May F, Lorenz V, et al.
Combined in vivo depletion of glycoprotein VI and C-type lectin-like receptor 2 severely compromises hemostasis and abrogates arterial thrombosis in mice. Arterioscler Thromb Vasc Biol. 2013;33(5):926-934.

12. Gros A, Syvannarath V, Lamrani L, et al. Single platelets seal neutrophil-induced vascular breaches via GPVI during immune-complex-mediated inflammation in mice. Blood. 2015;126(8):1017-1026.

13. Rayes J, Jadoui S, Lax S, et al. The contribution of platelet glycoprotein receptors to inflammatory bleeding prevention is stimulus and organ dependent. Haematologica. 2018;103(6):e256-e258.

14. Devi S, Kuligowski MP, Kwan RY, et al. Platelet recruitment to the inflamed glomerulus occurs via an alphaIlbbeta3/GPVI-dependent pathway. Am J Pathol. 2010;177(3):1131-1142.

15. Boilard E, Nigrovic PA, Larabee K, et al. Platelets amplify inflammation in arthritis via collagen-dependent microparticle production. Science. 2010;327(5965):580-583.

16. Pierre S, Linke B, Suo J, et al. GPVI and Thromboxane Receptor on Platelets Promote Proinflammatory Macrophage Phenotypes during Cutaneous Inflammation. J Invest Dermatol. 2017;137 (3):686-695.

17. Rayes J, Lax S, Wichaiyo S, et al. The podoplanin-CLEC-2 axis inhibits inflammation in sepsis. Nat Commun. 2017;8(1):2239.

18. Lax S, Rayes J, Wichaiyo S, et al. Platelet CLEC-2 protects against lung injury via effects of its ligand podoplanin on inflammatory alveolar macrophages in the mouse. Am J Physiol Lung Cell Mol Physiol. 2017;313(6):L1016-L1029.

19. Moreira CF, Cassini-Vieira P, da Silva MF, Barcelos LS. Skin Wound Healing Model Excisional Wounding and Assessment of Lesion Area. Bio-protocol. 2015;5(22): e1661.
20. Payne H, Ponomaryov T, Watson SP, Brill A. Mice with a deficiency in CLEC-2 are protected against deep vein thrombosis. Blood. 2017;129(14):2013-2020.

21. Mendonca RJ, Mauricio VB, Teixeira Lde B, Lachat JJ, Coutinho-Netto J. Increased vascular permeability, angiogenesis and wound healing induced by the serum of natural latex of the rubber tree Hevea brasiliensis. Phytother Res. 2010;24(5):764768.

22. Chen J, Kasper M, Heck T, et al. Tissue factor as a link between wounding and tissue repair. Diabetes. 2005;54(7):2143-2154.

23. Numata $Y$, Terui $T$, Okuyama $R$, et al. The accelerating effect of histamine on the cutaneous wound-healing process through the action of basic fibroblast growth factor. J Invest Dermatol. 2006;126(6):1403-1409.

24. McDonald DM, Baluk P. Significance of blood vessel leakiness in cancer. Cancer Res. 2002;62(18):5381-5385.

25. Dvorak HF. Tumors: wounds that do not heal-redux. Cancer Immunol Res. 2015;3 (1):1-11.

26. Yin T, He S, Liu X, et al. Extravascular red blood cells and hemoglobin promote tumor growth and therapeutic resistance as endogenous danger signals. J Immunol. 2015;194(1):429-437.

27. Kubo M, Van de Water L, Plantefaber LC, et al. Fibrinogen and fibrin are anti-adhesive for keratinocytes: a mechanism for fibrin eschar slough during wound repair. J Invest Dermatol. 2001;117(6):1369-1381.

28. Ronfard V, Barrandon Y. Migration of keratinocytes through tunnels of digested fibrin. Proc Natl Acad Sci U S A. 2001;98(8):4504-4509.

29. Geer DJ, Andreadis ST. A novel role of fibrin in epidermal healing: plasminogenmediated migration and selective detachment of differentiated keratinocytes. J Invest Dermatol. 2003;121(5):1210-1216.

30. Chalupowicz DG, Chowdhury ZA, Bach TL, Barsigian C, Martinez J. Fibrin II 
induces endothelial cell capillary tube formation. J Cell Biol. 1995;130(1):207-215.

31. Laurens N, Koolwijk P, de Maat MP. Fibrin structure and wound healing. J Thromb Haemost. 2006;4(5):932-939.

32. Sahni A, Francis CW. Vascular endothelial growth factor binds to fibrinogen and fibrin and stimulates endothelial cell proliferation. Blood. 2000;96(12):3772-3778.

33. Higazi AA, Barghouti, II, Ayesh SK, Mayer M, Matzner Y. Inhibition of neutrophil activation by fibrinogen. Inflammation. 1994;18(5):525-535.

34. Hanson AJ, Quinn MT. Effect of fibrin sealant composition on human neutrophil chemotaxis. J Biomed Mater Res. 2002;61 (3):474-481.

35. Dovi JV, He LK, DiPietro LA. Accelerated wound closure in neutrophil-depleted mice. J Leukoc Biol. 2003;73(4):448-455.

36. Mori R, Kondo T, Ohshima T, Ishida Y, Mukaida N. Accelerated wound healing in tumor necrosis factor receptor p55-defi- cient mice with reduced leukocyte infiltration. FASEB J. 2002;16(9):963-974.

37. Wong SL, Demers M, Martinod K, et al. Diabetes primes neutrophils to undergo NETosis, which impairs wound healing. Nat Med. 2015;21(7):815-819.

38. Stavrou EX, Fang C, Bane KL, et al. Factor XII and UPAR upregulate neutrophil functions to influence wound healing. J Clin Invest. 2018;128(3):944-959.

39. Wilgus TA, Roy S, McDaniel JC. Neutrophils and Wound Repair: Positive Actions and Negative Reactions. Adv Wound Care (New Rochelle). 2013;2(7):379-388.

40. Lishko VK, Burke T, Ugarova T Antiadhesive effect of fibrinogen: a safeguard for thrombus stability. Blood. 2007;109(4):1541-1549.

41. Crane MJ, Daley JM, van Houtte O, Brancato SK, Henry WL Jr, Albina JE. The monocyte to macrophage transition in the murine sterile wound. PLoS One. 2014; 9(1):e86660.
42. Francke A, Herold J, Weinert S, Strasser RH Braun-Dullaeus RC. Generation of mature murine monocytes from heterogeneous bone marrow and description of their properties. J Histochem Cytochem. 2011; 59(9):813-825.

43. Lucas T, Waisman A, Ranjan R, et al Differential roles of macrophages in diverse phases of skin repair. J Immunol. 2010, 184(7):3964-3977.

44. Macrae FL, Duval C, Papareddy P, et al. A fibrin biofilm covers blood clots and protects from microbial invasion. J Clin Invest. 2018;128(8):3356-3368

45. Kim MH, Liu W, Borjesson DL, et al Dynamics of neutrophil infiltration during cutaneous wound healing and infection using fluorescence imaging. J Invest Dermatol. 2008;128(7):1812-1820.

46. Lindholm C, Searle R. Wound management for the 21st century: combining effectiveness and efficiency. Int Wound J. 2016;13 Suppl 2:5-15. 